\title{
Erratum to: The Validity of Using Analogue Patients in Practitioner- Patient Communication Research: Systematic Review and Meta-Analysis
}

\author{
Liesbeth M. van Vliet, MSc ${ }^{7}$, Elsken van der Wall, MD, PhD', Akke Albada, $P h D^{7}$, \\ Peter M. M. Spreeuwenberg, MA ${ }^{7}$, William Verheul, $\mathrm{MSc}^{7}$, and Jozien M. Bensing, PhD ${ }^{7,3}$ \\ ${ }^{1}$ NIVEL (Netherlands Institute for Health Services Research), Utrecht, The Netherlands; ${ }^{2}$ University Medical Center Utrecht, Utrecht University, \\ Utrecht, The Netherlands; ${ }^{3}$ Department of Clinical and Health Psychology, Utrecht University, Utrecht, The Netherlands.
}

J Gen Intern Med 27(11):1570

DOI: $10.1007 / \mathrm{s} 11606-012-2215-1$

(c) Society of General Internal Medicine 2012

\section{Erratum to: $J$ Gen Intern Med \\ DOI: $10.1007 / \mathrm{s} 11606-012-2111-8$}

On page 2 of the original article, in line 7 , reference numbers $8-11$ are cited. Only 8 and 11 should be cited-the publisher regrets the error.

Headings were mistakenly omitted from the Abstract. The correct Abstract appears as follows.

\begin{abstract}
BACKGROUND: When studying the patient perspective on communication, some studies rely on analogue patients (patients and healthy subjects) who rate videotaped medical consultations while putting themselves in the shoes of the video-patient.

OBJECTIVE: To describe the rationales, methodology, and outcomes of studies using video-vignette designs in which videotaped medical consultations are watched and judged by analogue patients.
\end{abstract}

DATA SOURCES: Pubmed, Embase, Psychinfo and CINAHL databases were systematically searched up to February 2012.
DATA EXTRACTION: Data was extracted on: study characteristics and quality, design, rationales, internal and external validity, limitations and analogue patients' perceptions of studied communication. A meta-analysis was conducted on the distribution of analogue patients' evaluations of communication. RESULTS: Thirty-four studies were included, comprising both scripted and clinical studies, of average-to-superior quality. Studies provided unspecific, ethical as well as methodological rationales for conducting video-vignette studies with analogue patients. Scripted studies provided the most specific methodological rationales and tried the most to increase and test internal validity (e.g. by performing manipulation checks) and external validity (e.g. by determining identification with video-patient). Analogue patients' perceptions of communication largely overlap with clinical patients' perceptions. The meta-analysis revealed that analogue patients' evaluations of practitioners' communication are not subject to ceiling effects.

CONCLUSIONS: Analogue patients' evaluations of communication equaled clinical patients' perceptions, while overcoming ceiling effects. This implies that analogue patients can be included as proxies for clinical patients in studies on communication, taken some described precautions into account. Insights from this review may ease decisions about including analogue patients in video-vignette studies, improve the quality of these studies and increase knowledge on communication from the patient perspective.

Corresponding Author: Liesbeth M. van Vliet, MSc; NIVEL (Netherlands Institute for Health Services Research), PO box 15683500 BN, Utrecht, The Netherlands (e-mail:l.vanvliet@ nivel.nl).

The online version of the original article can be found at http:// dx.doi.org/10.1007/s11606-012-2111-8.

Published online September 26, 2012 